\title{
1 Calibration of Cherenkov Detectors for Monoenergetic 2 Photon Imaging in Active Interrogation Applications
}

P.B. Rose Jr. ${ }^{\mathrm{a}}$, A.S. Erickson ${ }^{\mathrm{a}, *}$

ar70 State St., Georgia Institute of Technology, Atlanta, Georgia 30332-0405

\begin{abstract}
Active interrogation of cargo containers using monoenergetic photons offers a rapid and low-dose approach to search for shielded special nuclear materials. Cherenkov detectors can be used for imaging of the cargo provided that gamma ray energies used in interrogation are well resolved, as the case in ${ }^{11} \mathrm{~B}(\mathrm{~d}, \mathrm{n}-$ $\gamma{ }^{12} \mathrm{C}$ reaction resulting in $4.4 \mathrm{MeV}$ and $15.1 \mathrm{MeV}$ photons. While an array of Cherenkov threshold detectors reduces low energy background from scatter while providing the ability of high contrast transmission imaging, thus confirming the presence of high-Z materials, these detectors require a special approach to energy calibration due to the lack of resolution. In this paper, we discuss the utility of Cherenkov detectors for active interrogation with monoenergetic photons as well as the results of computational and experimental studies of their energy calibration. The results of the studies with sources emitting monoenergetic photons as well as complex gamma ray spectrum sources, for example ${ }^{232} \mathrm{Th}$, show that calibration is possible as long as the energies of photons of interest are distinct.

Keywords: Cherenkov detectors, active interrogation, imaging, shielded special nuclear material
\end{abstract}

\section{Introduction}

Cargo screening using radiation monitors is an integral part of US safety and security strategy [1, especially when searching for illicit transportation of small volumes, on the order of $100 \mathrm{~cm}^{3}$, of special nuclear material (SNM).The

\footnotetext{
*Corresponding author, telephone: (+1) 404-385-0419

Email addresses: prose6@gatech.edu (P.B. Rose Jr.), anna.erickson@me.gatech.edu (A.S. Erickson)
} 
impracticality of physically opening and inspecting each air, ground, or sea container resulted in the development of a number of radiation-based scanning techniques, both passive [2] using the signatures of the material and active [3 5 ] with an external radiation source. Passive techniques have an advantage of low cost and relative simplicity as compared to active interrogation systems, but rely on naturally emitted radiation from SNM, which is relatively easy to conceal with proper shielding [6]. One of the major problems associated with active interrogation methods is dose delivered to the cargo and operators. An active interrogation system capable of producing monoenergetic photons, that are a product of low-energy nuclear reactions, for example ${ }^{11} \mathrm{~B}(\mathrm{~d}, \mathrm{n}-\gamma){ }^{12} \mathrm{C}[7-10$, can be utilized to penetrate the shielding and reveal the SNM inside while delivering dose that is significantly lower than the dose associated with bremsstrahlungbased systems. Coupling these high energy monoenergetic photons with an array of detectors enables the gamma ray transmission through the cargo for the production of a high contrast planar image as well as to study properties of the cargo specific to nuclear materials, for example delayed neutron and gamma emissions. A conceptual illustration of transmission imaging using an array of detectors is shown in Figure 1. In this paper, we discuss the results of computational and experimental studies of energy calibration performed with Cherenkov detectors designed specifically for coupling with an active interrogation system for gamma ray transmission imaging [11] The image produced is a measure of the atomic number, $\mathrm{Z}$, of the material for a rapid identification of heavy items in a cargo container.

\section{Description of the system}

\subsection{Source of monoenergetic photons}

Multiple low-energy nuclear reactions can produce high energy gamma rays [], but for the purposes of this paper we will focus on an extension of one system in particular [10, 12]. In this system, a compact accelerator is used to direct deuterons into a ${ }^{11} \mathrm{~B}$ target, resulting in ${ }^{11} \mathrm{~B}(\mathrm{~d}, \mathrm{n}-\gamma){ }^{12} \mathrm{C}$ reaction. This leaves 


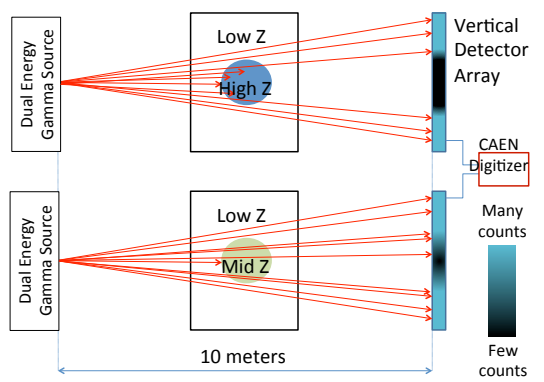

Figure 1: Conceptual design and principle of operation of imaging system based on monoenergetic photons

${ }_{56}{ }^{12} \mathrm{C}$ in excited states which produce $4.4 \mathrm{MeV}$ and $15.1 \mathrm{MeV}$ gamma rays at

${ }_{57}$ high intensity as well as less intense gamma rays during carbon's return to the ground state. By time gating the data acquisition with the accelerator pulse, it is possible to filter out undesired products from nuclear reactions caused by the emission of high energy neutrons and focuses on the fastest de-excitations of in Sheffield and Taducci [13].

The 4.4 MeV and 15.1 MeV gamma rays are used to produce a transmission image of the cargo, identifying relative $\mathrm{Z}$ of the material. The $4.4 \mathrm{MeV}$ photon interactions are heavily dominated by Compton scattering, therefore are a measure of the electron density of the material which is a function of the Z. The 15.1 MeV interactions are dominated by pair-production for mid- and high-Z materials. Cherenkov detectors are a part of the imaging approach of the system which provide high-frequency and low-background performance.

\subsection{Cherenkov detectors}

High radiation flux systems pose unique detection challenges which Cherenkov detectors are well suited for. High energy resolution is not a priority for this system since the $4.4 \mathrm{MeV}$ and $15.1 \mathrm{MeV}$ gamma rays are well separated. Such a system must be scalable, customizable, composed of inexpensive materials, resistant to background radiation, and capable of processing high interaction 
rates. Cherenkov light emission [14, 15] is a response of matter to the motion of a charged particles traveling at a speed exceeding the phase velocity of light in that medium. It is commonly produced in dielectric materials through Compton electrons or pair-production electrons and positrons. The intensity of light produced by this process is much less than that of luminescence (the basis for scintillation detector operation) requiring sensitive optical photon detection equipment such as a photo-multiplier tube (PMTs) or solid state silicon photomultiplier (SiPMs). On a positive side, typical scintillator detectors have a decay time measured in microseconds while Cherenkov radiation is nearly instantaneous and with fast pulse processing equipment can be measured in picoseconds. Other unique properties distinguishing Cherenkov radiation from luminescence are directionality, UV-concentrated photon spectrum, and energy threshold that is related to the dielectric constant of the chosen material.

Cherenkov radiation is emitted in a forward cone in the direction of the charged particle which is also forward peaked at high incident gamma ray energies (above $1 \mathrm{MeV}$ ). This cone is emitted in the direction of the PMT or other photon sensing device making the geometrical considerations of the detector crucial for peak performance. The inherent energy threshold properties of Cherenkov are based on the refractive index of a material which sets the phase velocity of the speed of light in that material. The refractive indices of materials can be finely tuned to produce an energy threshold rejecting particles below a certain energy suppressing background radiation. Significant background reduction focuses on the energies desired thus further reducing the demands on the detector allowing for faster scanning times. Figure 2 illustrates the relationship of the Cherenkov threshold to the energy of the incident gamma rays and subsequent Compton electrons.

Since there are neutrons present in the ${ }^{11} \mathrm{~B}(\mathrm{~d}, \mathrm{n}-\gamma){ }^{12} \mathrm{C}$ reaction, the Cherenkov radiator was chosen to be resistant to neutron activation and other neutron reactions such as the $H(n, \gamma) D$ capture reaction. These secondary reactions can introduce photons generated inside the detector adding massive overhead to the system and degrading the dual monoenergetic benefits of the initial nuclear re- 
action. After considering and testing many materials, Quartz, GE type 214 was selected as a final radiator option due to a very pure $\mathrm{SiO}_{2}$ crystalline lattice structure with excellent light transmission properties, especially in the UV part of the spectrum.

Quartz provides a unique set of material properties well suited to this application. It has a higher density than most other materials typically used for Cherenkov radiation, for example water or acrylic, allowing to use smaller detectors to match the photon interaction probability. Quartz is not hydrophilic like many scintillator crystals and is very resistant to thermal expansion in the anticipated operating temperature range, 0 to $40^{\circ} \mathrm{C}$. The internal light transmission is efficient due to high purity of material and high internal reflectivity, allowing more optical photons to reach the PMT. The geometry chosen for this source is rods $2.5 \mathrm{~cm}$ in diameter by $15.2 \mathrm{~cm}$ in length. To maximize optical photon transmission efficiency, the quartz is wrapped with PTFE tape reflector, then light proofed with a rubber heatshrink. This combination has proved to be a very efficient, rugged Cherenkov radiator design that is very resistant to neutron activation and can be used in the field under any weather conditions.

The absolute energy threshold for a detector made from this type of quartz, $\mathrm{SiO}_{2}$, is calculated to be $0.336 \mathrm{MeV}$. This threshold is calculated assuming maximum energy transfer in a Compton scattering event which is rarely the case, especially at lower energies. Signal generation will be very low until approximately $0.55 \mathrm{MeV}$, referred to here as effective threshold, where the partial energy

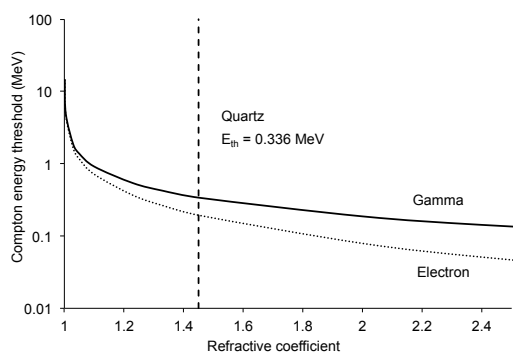

Figure 2: Natural threshold of Cherenkov radiation as a function of refractive index. 
transfer consistently imparts enough kinetic energy to the Compton electron to generate Cherenkov radiation. A large portion of background radiation is below this threshold and therefore will not produce any signal in the detector.

\section{Overview of the approach to energy calibration with Cherenkov detectors}

Energy calibration for scintillation detectors is a well-understood process that uses either the maximum or centroid of a peak in the energy histogram to derive the incident particle energy versus energy bin relationship. This method does not work for Cherenkov radiation since there are no well resolved peaks due to low levels of light emission for particles in MeV energy range. Instead, we must consider features in the energy histogram and relate them to the physics taking place. Using features such as the tail slopes, inflection points, and shoulder we can assign maximum energy deposition, most probable Compton electron energy corresponding to the energy of incident photon, and average energy deposited respectively.

In the detector calibration approach offered here, the primary source of particle information is the "tail" of the energy histogram as shown in Figure 3. In an ideal scenario, the location of the tail with respect to the energy bin axis will indicate the energy calibration point. However, very high flux situations

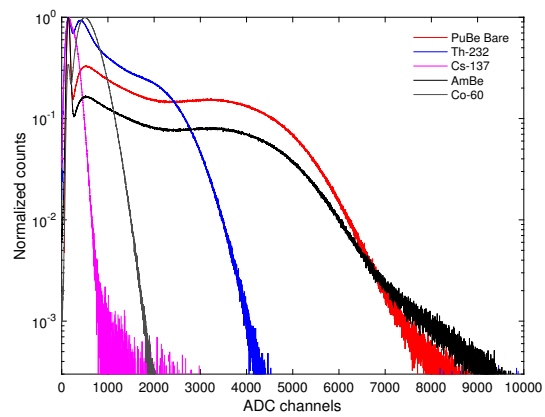

Figure 3: Cherenkov emission spectra associated with different sources. All data have been normalized to 1 to account for the differences in activity of these sources. 
produced by accelerators are far from ideal. The Cherenkov detectors were designed to work in such environments; although statistically rare due to the fast nature of the electromagnetic emission taking place, pulse pile-up and multiple particle interaction coincidences occur affecting the spectrum tail. Thus, the location where the tail meets the energy bin axis cannot be considered as the maximum energy transferred. When analyzing the tail of a Cherenkov spectrum generated in a high flux environment, we look at the inflection point of the tail where it appears as two linear relationships meet as shown in Figure 3 . Observing the pile-up effects in various experimental conditions and extrapolating the linear part of the spectrum tail, we choose to cut off everything below $0.2 \%$ of the total signal. This metric is specific to the detector and electronics and will vary for different systems. However, using this cutoff metric, we can assign the location where the energy histogram intersects the new $\mathrm{x}$-axis as the maximum energy deposited from the incident photon which provides the relationship for channel vs. incident particle energy needed for energy calibration. This relation, once determined for the detector, will hold for most sources, unless very high fluxes are experienced as compared to the reference system. To be consistent in assigning this point, we use an algorithm we designed to analyze the slope of the remaining tail and solve for the ADC channel where the linear relationship meets the cutoff. This algorithm takes into account the slope of the tail as well as the spread in the data to indicate event pile-up and coincidence.

\section{Modeling and simulation with Geant4}

The performance of the detectors has been characterized and optimized using Monte Carlo simulations. Geant4.10 was used to model the detector due to the capability of advanced optical photon transport, control of material properties, and detailed physics including Cherenkov radiation in particular. The Geant4 toolkit [16? ] provides a flexible framework for the simulation of particle transport and interaction with matter. The quality of the simulations relies heavily on user inputs for properties and physical processes of interest. 
When applied to Cherenkov detectors, Geant4 has the capability of triggering and tracking optical processes, which include the generation of Cherenkov photon by a charged particle. Rayleigh scattering, bulk absorption and reflectionrefraction media-boundary interactions are also part of the optical processes and are wavelength-dependent. Mechanical and optical properties of quartz from experimental measurements were combined with information provided by the manufactures including the efficiency and sensitivity of photomultiplier tubes. Modeling studies included the quartz crystal size and shape combined with a variety of PMTs to determine the optimal configuration for the given application.

Prior to detector construction for active interrogation applications, its efficiency has been studied as a function of gamma ray energy and the PMT properties assuming $100 \%$ geometric efficiency. The sources included ${ }^{137} \mathrm{Cs}$, ${ }^{60} \mathrm{Co},{ }^{232} \mathrm{Th}, \mathrm{PuBe}$, and AmBe, as available in the laboratory for experimental validation. AmBe and PuBe sources were used as a surrogate for the lower end of the deuteron-induced spectrum corresponding to the monoenergetic photons of 4.4 MeV. Both of these sources emit 4.4 MeV gamma rays from the same transition as the accelerator driven source, one of the excited states of carbon. Since the actual ${ }^{11} \mathrm{~B}(\mathrm{~d}, \mathrm{n}-\gamma){ }^{12} \mathrm{C}$ reaction also produces copious neutrons, hydrogenous materials were studied in order to understand the shielding needs of experimental accelerator-driven reactions. AmBe and PuBe sources were combined with acrylic or poly cylinders producing neutron capture by hydrogen resulting in a $2.2 \mathrm{MeV}$ gamma ray. Materials rich in hydrogen that will be used to shield neutrons during the active interrogation will create a significant contribution to the signal due to the capture reaction. By including the neutron in the simulation, an accurate estimate of the lower end of the anticipated spectrum is obtained while also allowing for preliminary validation and transmission experiments to be carried out. The summary of the simulated efficiency for the four sources is shown in Table 1. This metric is the theoretical maximum as it counts even single photoelectrons generated in the PMT. In experiment, some of the signal will be filtered out as dark current in the PMT. 
Table 1: Detector efficiency by source

\begin{tabular}{|l||c|c|c|}
\hline Source & Photon energy (keV) & 2" long radiator & 4" long radiator \\
\hline${ }^{137} \mathrm{Cs}$ & 662 & $19.6 \%$ & $25.4 \%$ \\
${ }^{60} \mathrm{Co}$ & 1173 and 1332 & $33.9 \%$ & $51.5 \%$ \\
${ }^{232} \mathrm{Th}$ & 2614 (maximum) & $30.8 \%$ & $49.1 \%$ \\
$\mathrm{PuBe}$ & 4440 & $25.8 \%$ & $45.8 \%$ \\
\hline
\end{tabular}

The detector efficiency is dependent on the energy of the photon as well as the length of the detector. Most scintillating detectors have a higher efficiency with ${ }^{137} \mathrm{Cs}$ (662 keV gamma ray) than they do at higher energy sources which is not the case for Cherenkov detectors. The effective energy threshold for photon-generated signal of these detectors is about $550 \mathrm{keV}$, meaning the electron must receive a large majority of the energy during the Compton scatter. The scattering angle probability is closer to isotropic than forward peaked at this energy as predicted by the Klein-Nishina formula. This means that most Compton interactions will not transfer enough energy to the electron to generate Cherenkov radiation. The relationship between photoelectrons produced in the PMT and the incident particle energy can be deduced similarly to the Cherenkov detector method introduced earlier. However, since the simulation is sequential, there is no pile-up or multi-particle coincidence to be observed. The highest number of photoelectrons produced corresponds to the maximum energy transfer to the Cherenkov radiating material. To ensure we are considering statistically relevant results as well as keeping the simulation consistent with experimental studies, we choose the $0.2 \%$ cutoff for the energy calibration. Considering the physics behind the interactions, the total energy deposited is less than the energy of the photon emitted by the source. However, since this is common across all interactions in the energy range considered, the relation between the photon energy can be established as shown in Figure 4

The points on the plot represent the maximum gamma ray energies of the 


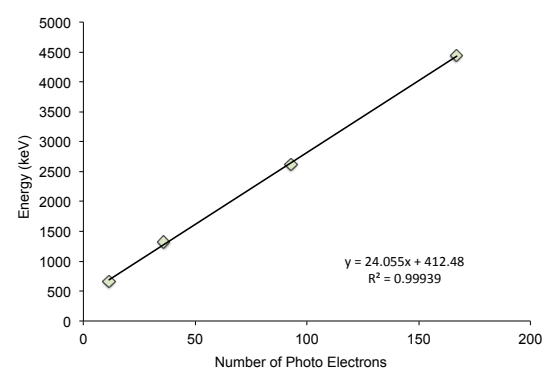

Figure 4: Simulated energy calibration of the 2-inch long detector

sources used from low to high: ${ }^{137} \mathrm{Cs},{ }^{60} \mathrm{Co},{ }^{232} \mathrm{Th}$, and $\mathrm{PuBe}$, respectively. The results are shown for the 2-inch long detector, and the 4-inch long design displays a nearly identical relationship. The simulation predicts a highly linear relation between the energy deposited in the detector and the number of photoelectrons created in the PMT across this incident photon energy range. These relationships are used to produce the energy calibrated histograms for the 2-inch long detector fabricated for experimental testing as shown in Figure 5 It should be noted that additional studies are underway to include $15.1 \mathrm{MeV}$ gamma rays for the future analysis. Due to higher energy of the gamma ray, the interactions are a combination of Compton scattering and pair production which may lead to deviation from linearity observed in Figure 4. The x-axis is the number of photoelectrons produced calibrated to incident photon energy in the R292 PMT, which has high UV sensitivity. This curve is not exactly what is expected experimentally because the photoelectrons have some energy distribution to them where the simulation assumes a constant energy.

\section{Experimental results}

Multiple detector designs have been built and tested including polymethyl methacrylate (PMMA) and quartz to understand detector properties as a function of gamma ray energy and detector length as well as for computational model validation. An array of detector samples is shown in Figure 6. The final validation was done with two quartz detectors of slightly different designs: a 2-inch 


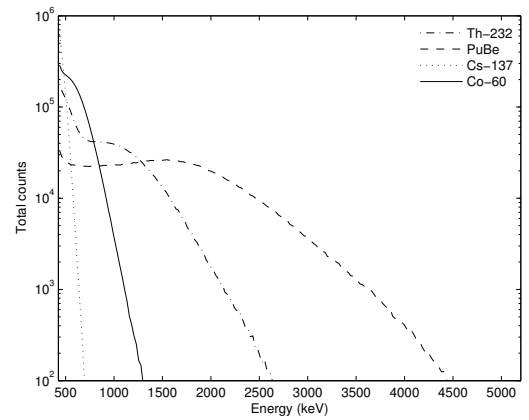

Figure 5: Calibrated spectral response of the 2-inch long detector (simulation)

long crystal coupled with a Hamamatsu R292 PMT and a 4-inch long crystal coupled with a R6095 PMT. Experimental energy calibration is more convoluted than computational. Statistically, event pile-up and multiplicity may become an issue even though Cherenkov radiation is a nearly instantaneous response to the Compton electron; however, the effects are minimized when compared to scintillators. As described in the calibration method, the first step is to apply a cut to the normalized counts, $0.2 \%$ of the total signal in this case, to subtract the contribution of noise. This also cuts off the signal from addition of two gamma rays as well as muon interactions which are much less probable than the photon interaction.

Even though the size of the detector is small, an occasional muon interaction deposits a large amount of energy in the detectors and can cause excess signal in any energy bin. Next, the slopes of the tails are analyzed, and the location where they meet the $\mathrm{x}$-axis (ADC channels) is correlated to the channel of full energy deposition (photopeak). These intersections are related to the maximum gamma energy emitted from the source. Figure 7 shows the resulting energy calibration curve for the 2-inch long detector. Even though the results are pedestal-subtracted, the nonlinearity of Cherenkov light generation near the threshold region precludes low-energy photons from being used in calibration. Photon energy of $661.7 \mathrm{keV}$ corresponding to ${ }^{137} \mathrm{Cs}$ is the lowest energy used.

Similarly to the computational results, the experiments show a strong linear 
relationship between energy deposited in the detector and the ADC channel. This trend line equation is used to convert the experimental data from ADC channels to energy of incident photons. The 2-inch long detector produced a very similar relationship and an equation for the unique energy calibration. The resulting energy calibration can be seen in Figure 8 for the 2-inch long detector.

When considering the Cherenkov spectra, it is evident that there are no wellresolved peaks produced by the detector. Instead, spectral information can be gathered from slopes of the tails and location of changes in slope, shoulders, and the data. In the lower energy range, there is little usable data. The peaks and shoulders that are most evident are related to the average energy transferred to the Compton electron in a scattering event. The average energy transferred to a Compton electron in a $4.4 \mathrm{MeV}$ scattering event from the PuBe/AmBe source is about $2.7 \mathrm{MeV}$. This is where the "peak" can be seen in Figure 8 in the PuBe spectrum. It is important to note the effective Cherenkov radiation energy threshold for a photon in this material is about $550 \mathrm{keV}$, and the linearity of the energy calibration breaks down as this limit is approached. Performing an energy calibration based on the three higher energy sources $\left({ }^{60} \mathrm{Co},{ }^{232} \mathrm{Th}\right.$, and $\mathrm{PuBe}$ ) yields a more accurate calibration among those energies, but the 662 $\mathrm{keV}$ from the ${ }^{137} \mathrm{Cs}$ virtually disappears from the plot. For later experiments including $15.1 \mathrm{MeV}$ photons, the $662 \mathrm{keV}$ will be omitted from the energy calibration.

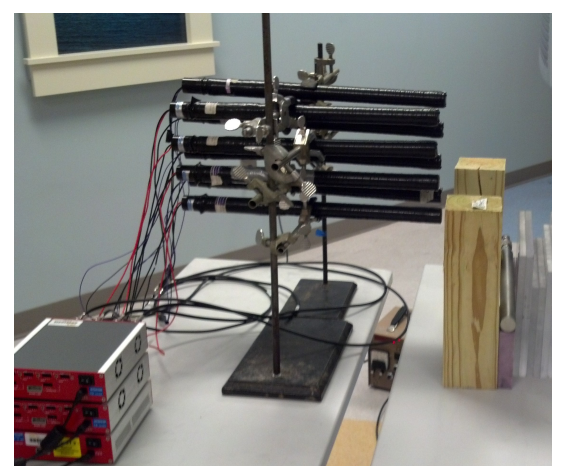

Figure 6: Experimental setup of various Cherenkov detectors. 


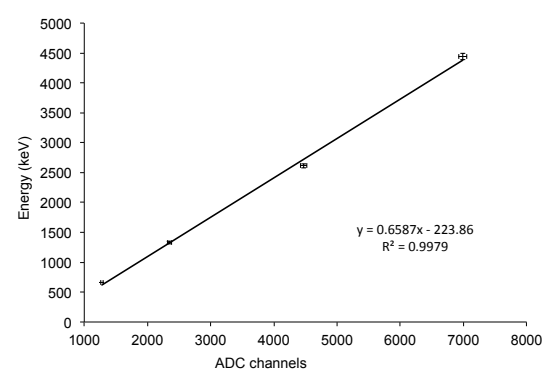

Figure 7: Experimental energy calibration of the 4-inch long detector

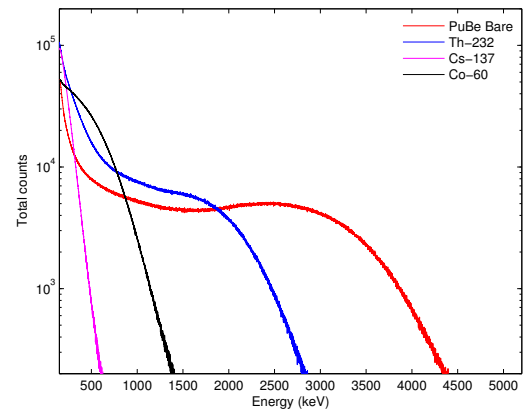

Figure 8: Calibrated spectral response of the 2-inch long detector (experimental)

The 4-inch long detector uses the R6095 which operates on a very different design than the traditional box-and-grid style R292 used in the 2-inch long detector. The R292 is more efficient when considering total photoelectrons created vs. photoelectron collected, but the electron transit time is much longer, and the pulses are stretched over a longer time period. This effect is on the order of $100 \mathrm{~ns}$ per pulse, where the pulse from the R6095 can be accepted in about 50 ns. Figure 9 shows the same experiments performed with the 4-inch long detector with the energy calibration performed by the same method.

Due to more favorable time properties of the R6095 PMT, the energy calibration for the 4-inch long detector appears to be more accurate than the 2-inch long detector. Another characteristic to notice is the formation of peaks in the region less than $1 \mathrm{MeV}$. These peaks are misleading because they are formed due to the less efficient photoelectron collection of R6095 PMT type. When 


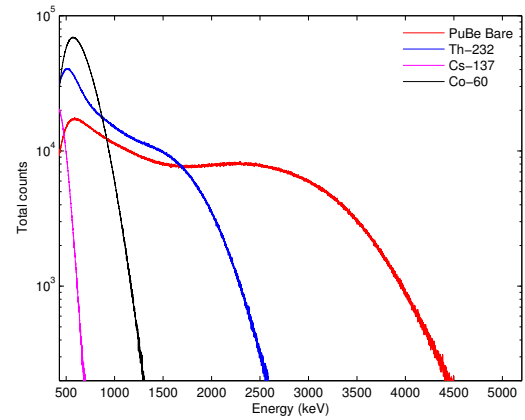

Figure 9: Energy calibrated experimental data from the 4-inch long detector.

few photoelectrons are produced, a large percentage could be lost at each stage. The more photoelectrons generated in the first stages, the more consistent the signal is. Even though the 4-inch long detector is much longer, it registers fewer counts for the $662 \mathrm{keV}$ photons than the 2-inch long detector did at the same distance from the source. The ratio of the maximum efficiency to the ${ }^{137} \mathrm{Cs}$ efficiency for each detector is much lower for the 4-inch long due to the PMT type. However, it is possible to see the difference between the combined peak from ${ }^{60} \mathrm{Co}$ (1.17 and $1.33 \mathrm{MeV}$ gamma rays) and ${ }^{232} \mathrm{Th}(0.911$ and $0.969 \mathrm{MeV}$ gamma rays).

The PuBe and AmBe sources were used with and without hydrogenous material present around the sources. This produces a significant number of 2.2 $\mathrm{MeV}$ gamma rays from the $(\mathrm{n}, \gamma)$ reaction on hydrogen. Figure 10 shows the experimental results of detection of gamma rays from an AmBe source with and without large poly cylinders beside the source. At the maximum gamma ray energy of $4.4 \mathrm{MeV}$, the two spectra line up very well. The relative contribution of the $2.2 \mathrm{MeV}$ gamma rays is very apparent in the run with polyethylene present. This mimics the low end of the accelerator driven source very closely. The average energy transferred to a Compton electron in a $2.2 \mathrm{MeV}$ scattering event is about $1.3 \mathrm{MeV}$ which is where the shoulder can be seen in the data where the poly is present. Figure 11 illustrates the potential technique of Cherenkov detector energy calibration. The terminal energy (ADC bin or computational 


\section{source}

Figure 10: Comparison of moderated and unmoderated experimental results from AmBe

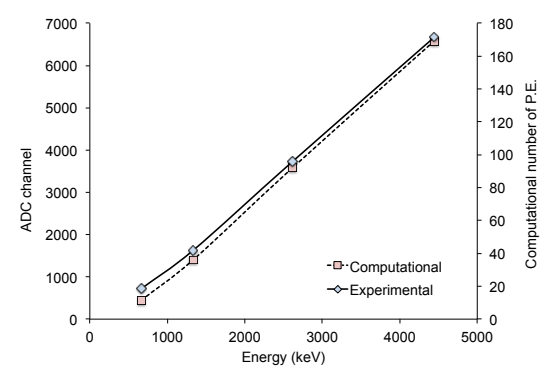

Figure 11: Correlation of simulation and experimental energy calibration for 2-inch long detector

\section{Conclusions}

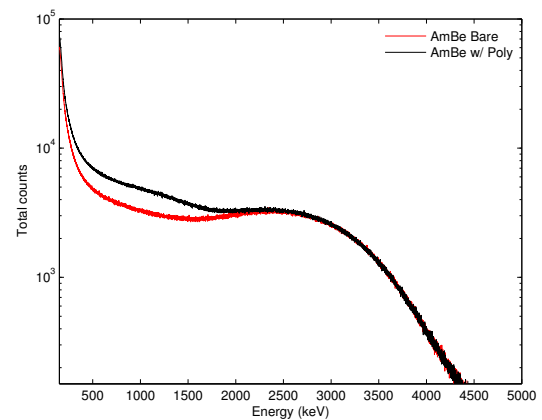

Two quartz detector designs were designed, optimized using Geant4 modeling and simulations, fabricated, and tested with various sources to validate 
the simulation predictions. The computational and experimental results were calibrated using a linear relationship of photon energy and photoelectrons or bins, respectively. The range of incident energies shows good linearity once the energy is sufficiently above the threshold. However, it is expected that above some energy, dependent upon the material, the linearity will break down. Once the $15.1 \mathrm{MeV}$ photons are introduced to the testing, the energy calibration will be tested for other relations, for example quadratic, as well as exclude the ${ }^{137} \mathrm{Cs}$ for improved accuracy.

These detectors will further be tested with an ion accelerator where the high energy monoenergetic photons can be produced via the nuclear reactions. Upon characterization of the signal generated from the high energy photons, the energy calibration method discussed here will be applied. After that, transmission studies through various areal densities atomic numbers will be completed in preparation to apply a large scale array of these detectors for cargo imaging purposes. This energy calibration technique opens the door to spectrum deconvolution, unfolding, of Cherenkov detectors. The process has been well proven with scintillators but not with Cherenkov detectors. Using information such as the slopes of the tails and shoulders as related to energies it is in theory possible to perform such calculations. More studies are on the way to understand the behavior of Cherenkov detectors in the environment with significant scattering as well as when several photon energies that are close together, for example $12.7 \mathrm{MeV}$ and $15.1 \mathrm{MeV}$ gamma rays associated with another proposed reaction ${ }^{12} \mathrm{C}(\mathrm{p}, \mathrm{p} / \gamma)$, are present.

\section{Acknowledgements}

This material is based upon work supported by the National Science Foundation under Grant No. ECCS-1348366 and by the U.S. Department of Homeland Security under Grant Award Number 2014-DN-077-ARI079-02. The views and conclusions contained in this document are those of the authors and should not be interpreted as necessarily representing the official policies, either expressed 
or implied, of the U.S. Department of Homeland Security. This work is a part of the ARI collaboration with Massachusetts Institute of Technology (R. Lanza) and Pennsylvania State University (I. Jovanovic).

\section{References}

[1] W. Schubert, On the security of our seaports, statement before the Committee on the Judiciary Subcommittee on Technology, Terrorism, and Government Information United States Senate (February 26 2002).

URL http://www.gpo.gov/fdsys/pkg/CHRG-107shrg85082/html/ CHRG-107shrg85082.htm

[2] K. Ziock, W. Craig, L. Fabris, et al., Large area imaging detector for longrange, passive detection of fissile material, IEEE Trans. Nucl. Sci. 51 (2004) $2238-2244$.

[3] J. Jones, D. Norman, K. Haskell, et al., Detection of shielded nuclear material in a cargo container, Nucl. Instrum. Meth. A 562 (2006) 1085-1088.

[4] J. Jones, B. Blackburn, S. Watson, et al., High-energy photon interrogation for non proliferation applications, Nucl. Instrum. Meth. B 261 (2007) 326330.

[5] R. C. Runkle, D. L. Chichester, S. J. Thompson, Rattling nucleons: New developments in active interrogation of special nuclear material, Nucl. Instrum. Meth. A 663 (2012) 75-95.

[6] D. L. Chichester, E. H. Seabury, Active neutron interrogation to detect shielded fissionable material, in: International Topical Meeting on Nuclear Research Applications and Utilization of Accelerators, Vol. SM/EN-02, 2009.

[7] A. J. Antolak, B. L. Doyle, K. N. Leung, et al., Active interrogation using low-energy nuclear reactions, in: SPIE: Penetrating Radiation Systems and Applications VII, Vol. 5923, 2005, pp. 1-9. 
[8] B. Ludewigt, A. Antolak, E. Henestroza, et al., Coaxial mono-energetic gamma generator for active interrogation, in: AIP Conference Proceedings: CAARI2008, 2008.

[9] M. Brandis, V. Dangendorf, C. Piel, et al., Nuclear reaction based radiation source for explosives and snm detection in massive cargo, in: AIP Conference Proceedings, Vol. 1336, 2005, pp. 711-716.

[10] S. Kurennoy, R. Garnett, L. Rybarcyk, Intense combined source of neutrons and photons for interrogation based on compact deuteron rf accelerator, in: 23rd Conference on Application of Accelerators in Research and Industry, CAARI 2014, 2014.

[11] P. B. Rose Jr., A. Erickson, Detection of shielded special nuclear material using high energy gamma ray transmission imaging and cherenkov detectors, in: INMM Annual Meeting, 2014.

[12] R. C. Lanza, A. Erickson, J. Fisher, et al., Nuclear reaction based monoenergetic gamma ray radiography system for detection of nuclear materials, in: Nuclear Science Symposium and Medical Imaging Conference (NSS/MIC), 2014.

[13] T. Taddeucci, R. Sheffield, Neutron and gamma-ray production with lowenergy beams, Tech. Rep. LA-UR-07-2724, Los Alamos National Laboratory (2007).

[14] P. Cherenkov, Vidimoe svechenie chistykh zhidkostei pod deistviem $\gamma$ radiatsii, Dokl. Akad. Nauk SSSR 2 (1934) 451.

[15] P. Cherenkov, Visible radiation produced by electrons moving in a medium with velocities exceeding that of light, Phys. Rev 52 (1937) 378-379.

[16] J. Allison, et al., Geant4 developments and applications, in: IEEE Trans. Nucl. Sci., Vol. 53, 2006, pp. 270-278. 